\title{
A Close Association of Three Carbon Stars in the Direction of M92
}

\author{
O. M. KURTANIDZE and M. G. NIKOLASHVILI \\ Abastumani Astrophysical Observatory \\ 383762 Abastumani, Republic of Georgia
}

The discovery of the first faint $(V>15.0)$ high-latitude carbon star (FHLCS) was announced by Sanduleak (1980, PASP, 92, 246). It is located in the direction of the Magellanic Stream. On low-dispersion spectral plates $\left(1250 \AA \mathrm{mm}^{-1}\right.$ at $\left.\mathrm{H}_{\gamma}, \mathrm{IIIa}-\mathrm{J}, \mathrm{F}\right)$ taken with the 70 -cm meniscus telescope for identification of blue horizontal-branch stars in the globular cluster M92, two FHLCS were accidentally discovered (Kurtanidze 1980, Astron. Tsirk., 1109, 3) near the known bright carbon star HD $156074=$ Ste 3795 . The data on these stars are tabulated below along with data for two other faint carbon stars found in the field of the cluster of galaxies A2199. Slit spectra of the two new C stars were obtained by P. Green (1995, private communication). The

\begin{tabular}{rcrccl}
\hline \multicolumn{1}{c}{$\alpha_{2000}$} & \multicolumn{1}{c}{$\delta_{2000}$} & $V$ & $B-V$ & $V-R$ & Notes \\
\hline 161821.3 & 392320 & & & & New \\
163920.2 & 343123 & & & & SP 105 \\
171331.2 & 420623 & 7.80 & & & Ste 3795 \\
171411.8 & 420031 & 14.60 & 1.14 & 1.16 & New \\
171457.5 & 421024 & 15.98 & 2.22 & 1.22 & Ste 3801 \\
\hline
\end{tabular}

surface density of $\mathrm{C}$ stars in this region is equal to 30 per sq. deg., although their mean surface density is only about $0.03 \mathrm{C}$ star per sq. deg. at $|b|>30^{\circ}$. The proper motions of the faint carbon stars have been determined from the digitized POSS-I, II surveys. None of these four faint objects shows detectable proper motion (MacConnell 1996, private communication). The astrometric and photometric data were obtained from plates taken with 2-m Tautenburg Schmidt (1961-1990) and the 0.7-m Abastumani meniscus telescope (19781989). 